\title{
Improved model for secondary neutron production in nucleus-nucleus collision at intermediate energies
}

\author{
L.W. Townsend ${ }^{1}$, S. Sriprisan ${ }^{1, a}$, and F.A. Cucinotta ${ }^{2}$ \\ 1 Department of Nuclear Engineering, University of Tennessee, Knoxville TN 37996-2300, USA \\ 2 NASA Lyndon B. Johnson Space Center, Houston, TX 77058, USA
}

\begin{abstract}
In previous work an analytical knockout-ablation coalescence model capable of making quantitative predictions of the neutron spectra from high-energy nucleon-nucleus and nucleus-nucleus collision has been developed. The physics of the knockout-ablation model is similar to that in intranuclear cascade codes using Monte Carlo methods. In the first or knockout stage, the collision between two nuclei results in one or more nucleons being knocked out of the projectile and/or the target nucleus. This results in an excited nucleus (called a prefragment), which is lighter than the original species. This excited prefragment decays to the ground state by emitting one or more nucleons, composites (such as deuterons or alphas) and gammas. This is the ablation (second) stage of the reaction. Significant improvements in model predictions were made previously by incorporating important coalescence effects into the formalism. Coalescence occurs when particles with similar momenta located close together in space recombine to form heavier nuclei, such as deuterons, ${ }^{3} \mathrm{H}$ and ${ }^{3} \mathrm{He}$. In this work the important effects of alpha particle coalescence are included. The improved theory is described and results for predicted neutron and light ion energy and angular spectra are presented and compared with published measurements of secondary neutron and light ion production for a variety of collision pairs.
\end{abstract}

\section{Introduction}

Secondary neutron production from heavy-ion fragmentation processes in nucleus-nucleus collisions is currently a relevant topic of both an experimental and a theoretical research for a variety of applications. Fragmentation of heavy-ions in collisions is often described using a two-step process: the abrasion-ablation model. In the first step, the collision of two nuclei results in nucleons being knocked out of an interaction region. The size of this overlap region depends on the impact parameter of the collision. In the second stage, the remaining prefragment nucleus is highly excited and continues to travel with its pre-collision trajectory. The prefragment then decays by particle emissions to more stable configuration. A recent version of an abrasion-ablation model [1] was formulated to include the momentum distribution for nucleon production in heavy-ion collisions. In its original form, the formulation included knockout contributions from both projectile and target nuclei, but contributions only from the projectile nucleus for the ablation stage. Coalescence effects were also not included. In later work, the important effects of particle coalescence were partially incorporated [2] and the ablation stage was extended to include the target contributions. The basic assumption of coalescence is that during the knockout stage there are some nucleons that share the same volume and momentum space and they interact with each other to form heavier and more complex particles such as deuterons, tritons, helions, and alphas [2-7] to which the excess momentum and energies are transferred. Awes et al. [7], for example, presented comparisons of deuteron cross sections detected from the collisions of ${ }^{16} \mathrm{O}+{ }^{238} \mathrm{U}$ with the spectra predicted by using the coalescence model with the Coulomb repulsion from

${ }^{a}$ Presenting author, e-mail: isripris@utk.edu the target nucleus. The study showed the strong agreement between the measurements and the predictions especially in the forward angles. The work herein reported is largely based on the coalescence model described by Awes et al. [7].

The knockout-ablation-coalescence model presented herein extends the coalescence model to include alpha emission. Results for predicted secondary neutron energy and spectra are presented and compared with recently published measurements of secondary neutron production for a variety of collision pairs. Sample results for light ion production from coalescence are also presented.

\section{Neutron production model}

The underlying physical concepts applicable to the abrasionablation process have been discussed in details in refs. [1-6]. In this work, the brief outline of the theoretical model for predicting cross sections are described in the following subsections. The model is based upon an optical potential approximation to quantum mechanical multiple scattering theory.

\subsection{Knockout - ablation cross sections}

In the knockout-ablation formalism, neutrons are produced in both stages of the reaction. The neutron momentum distribution is given by

$$
\left(\frac{d \sigma}{d k}\right)_{\text {total }}=\left(\frac{d \sigma}{d k}\right)_{k o}+\left(\frac{d \sigma}{d k}\right)_{a b l}
$$

where $k$ is the wave number of the emitted neutrons. In the following discussion, it is assumed that neutrons are produced 
from the projectile. Neutron production from the target is obtained by interchanging the projectile and target nuclei in the formalism.

A simple expression for the abraded cross sections is found using the Glauber multiple scattering formalism as

$$
\left(\frac{d \sigma}{d k}\right)_{k o}=\sum_{n}\left(\begin{array}{l}
A_{p} \\
n
\end{array}\right) \int d^{2} b P^{A_{p}-n}(b) \frac{d N_{n}}{d k}
$$

where $A_{p}$ is the projectile mass number, $P(b)$ is the probability as a function of impact parameter that a nucleon is not removed in the knockout stage of the collision, and the momentum distribution of abraded particles is given by

$$
\left(\frac{d N_{n}}{d k}\right)=\frac{d N_{1}}{d k}[1-P(b)]^{n-1}
$$

where

$$
\int \frac{d N_{1}}{d k} d k=1-P(b) .
$$

In the optical limit, the probability becomes

$$
P(b)=\operatorname{Exp}\left[-\frac{2 i \chi(b)}{A_{p}}\right]
$$

where the eikonal phase $\chi$ is given in terms of one-body form factors $F(q)$ for the projectile and target and the nucleon scattering amplitude $f_{N N}$ as

$$
\chi(b)=\frac{A_{p} A_{T}}{2 \pi k_{N N}} \int d^{2} q \operatorname{Exp}(i q \bullet b) F_{p}(q) F_{T}(q) f_{N N}(q)
$$

with $A_{T}$ as the target mass number and $q$ is the momentum transfer in the collision. The form factor $F(q)$ is the Fourier transform of the appropriate (projectile or target) nuclear density.

After the projectile-target collision, the prefragment nuclei remain in various excited energy states. The decay of the excited prefragment occurs by the emission of one or more nucleons, composite particles, or gamma rays. To describe the production of neutrons from the decays of these excited prefragment, a Weisskopf-Ewing statistical decay model is used. Details are presented in ref. [1]. The parameter $P_{n}(j, k)$ is defined as the probability that a prefragment labeled $j$ with mass number $A_{j}$, charge number $Z_{j}$, and excitation energy $E_{j}^{*}$, emits a neutron of momentum $k$. The momentum distribution for neutron production for ablation in the projectile rest frame is then given by:

$$
\left(\frac{d \sigma}{d k}\right)_{a b l}=\sum_{j} \sigma_{k o}\left(A_{j}, Z_{j}, E_{j}^{*}\right) P_{n}(j, k)
$$

The neutron momentum distribution in the laboratory reference frame is found by multiplying equation (7) by the appropriate neutron energy to form a Lorentz invariant and then transforming to the laboratory system.

To simplify the calculation, the prefragment nucleus is assumed to be infinitely heavy and the energy emission spectrum is assumed to be isotropic in the emitting nucleus rest frame. The probability is

$$
P_{n}\left(j, E_{n}\right)=\frac{2 \mu_{n} g_{n} E_{n} \sigma_{C N} w_{0}\left(E_{j}^{*}-E_{n}\right)}{\sum_{l} F_{l}}
$$

where $\mu_{n}$ is the reduced mass, $g_{n}$ is the statistical weight, $\sigma_{C N}$ is the formation cross section by the inverse process, $w_{0}$ is the level density of the residual nucleus, and

$$
F_{l}=\int_{0}^{E_{l}^{*}-s_{j}} P_{l}(j, E) d E .
$$

If there is sufficient excitation energy available, then several particles will be emitted. Competition between the emission of particles such as $\mathrm{n}, \mathrm{p},{ }^{2} \mathrm{H},{ }^{3} \mathrm{H},{ }^{3} \mathrm{He}$ and $\alpha$ is permitted.

\subsection{The coalescence model}

In some cases a pair of particles, spatially near each other, and with similar momenta will coalesce to form heavier nuclei such as ${ }^{2} \mathrm{H},{ }^{3} \mathrm{H},{ }^{3} \mathrm{He}$ and $\alpha$. In this work we use a coalescence model adapted from the work of Awes [7]. The probability of finding a nucleon in the coalescence volume centered at a momentum $p$ is given by the product of the volume with the single nucleon momentum density:

$$
P=\frac{4 \pi}{3} p_{0}^{3} \frac{\gamma}{\bar{m}} \frac{d^{3} N(p)}{d p^{3}}
$$

where $d^{3} N(p) / d p^{3}$ represents the triple differential nucleon multiplicity, $\bar{m}$ is the average nucleon multiplicity, and $\gamma=$ $1+T / m$, where $\mathrm{T}$ is the kinetic energy per nucleon and $m$ is the nucleon mass. Using a Poisson multiplicity distribution, the probability of forming a composite particle with the momentum per nucleon $\vec{p}$ is

$$
\langle P(N, Z)\rangle=\frac{\left(\bar{m}_{Z} P_{Z}\right)^{Z}}{Z !} \frac{\left(\bar{m}_{N} P_{N}\right)^{N}}{N !}
$$

where we have assumed that the probabilities for the observation of neutrons $P_{N}$ and protons $P_{Z}$ are independent. Assuming the neutron distributions have the same shape as the proton distributions but are weighted by the $N / Z$ ratio of the composite system

$$
\frac{d^{3} N(0,1)}{d p^{3}}=\left(\frac{Z_{t}+Z_{p}}{N_{t}+N_{p}}\right) \frac{d^{3} N(1,0)}{d p^{3}} .
$$

Hence, the composite particle momentum distribution in the form of the coalescence relation used at relativistic energies is

$$
\frac{d^{3} N(Z, N)}{d p^{3}}=\left(\frac{Z_{t}+Z_{p}}{N_{t}+N_{p}}\right)^{Z} \frac{1}{N ! Z !}\left(\frac{4 \pi}{3} p_{0}^{3} \gamma\right)^{A-1}\left(\frac{d^{3} N(0,1)}{d p^{3}}\right)^{A}
$$

where $Z, N$, and $A$ are the composite particle charge, neutron number and mass, respectively; $N_{p}$ and $N_{t}$ are the projectile and target neutron numbers; and $Z_{p}$ and $Z_{t}$ are the projectile 


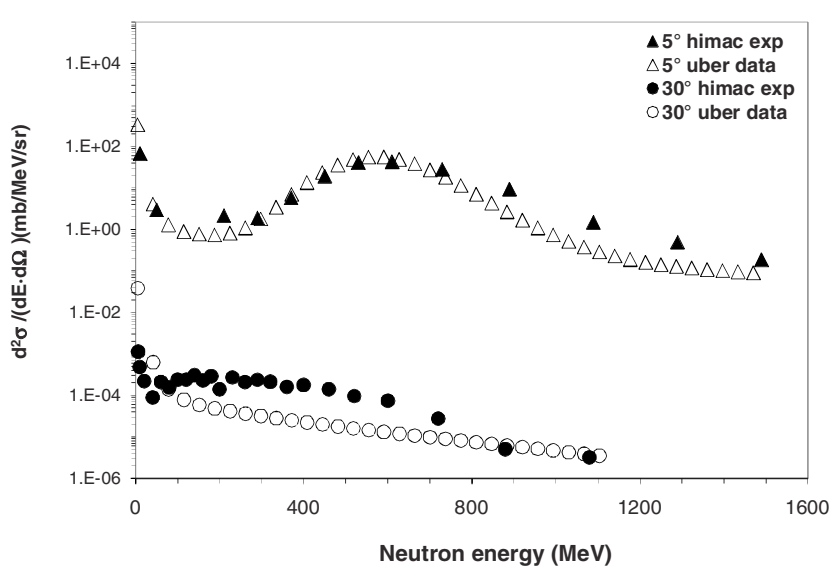

Fig. 1. Comparisons between calculated and measured neutron production cross sections at $5^{\circ}$ in the laboratory for the ${ }^{20} \mathrm{Ne}+{ }^{12} \mathrm{C}$ reaction at $600 \mathrm{~A} . \mathrm{MeV}$. The solid triangles and circles represent experimental data reported in ref. [9]. The open triangles and circles are the predictions from the model.

and target charge numbers. Details can be found in ref. [1]. The multiplicity is related to the momentum distribution by

$$
\frac{d^{3} N}{d p^{3}}=\frac{1}{\sigma_{0}} \frac{d^{3} \sigma}{d p^{3}}
$$

where $\sigma_{0}$ is the projectile-target total reaction cross section.

To transform the neutron cross section predictions between different reference frames, the invariant neutron cross section $\sigma_{I}(n)$ is introduced. The invariant cross section is given by $\frac{d^{3} \sigma}{d p^{3}}=\frac{1}{E^{*}} \sigma_{I}$.

Hence, the invariant neutron cross section becomes

$$
\sigma_{I}(Z, N)=C_{T}\left(\sigma_{I}(n)\right)^{A}
$$

where

$$
C_{T}=\left(\frac{Z_{t}+Z_{p}}{N_{t}+N_{p}}\right)^{Z} \frac{A}{N ! Z !}\left(\frac{4 \pi p_{0}^{3} \gamma}{3 \sigma_{0} m}\right)^{A-1}
$$

The critical radius $p_{0}$ within the coalescence region is treated as a free parameter. A wide range of values for $p_{0}$ have been proposed. For the purpose of comparison, the results in this work are presented based on the calculation with values of $p_{0}$ described in Awes et al. [7] and Nagamiya et al. [8].

\section{Results}

To illustrate the model, the production of secondary neutrons and light ions from $600 \mathrm{~A} \cdot \mathrm{MeV}{ }^{20} \mathrm{Ne}+{ }^{12} \mathrm{C}$ and $800 \mathrm{~A} . \mathrm{MeV}$ ${ }^{12} \mathrm{C}+{ }^{12} \mathrm{C}$ reactions have been calculated and their energy spectra and angular distributions compared with published data $[8,9]$. The energy dependent double differential cross section for neutrons traveling in the laboratory reference frame for ${ }^{20} \mathrm{Ne}$ beam at $5^{\circ}$ and $30^{\circ}$ angles are plotted in figure 1. The comparison between these data with predictions based on this formalism for the small forward angles, such as $5^{\circ}$, shows excellent agreement. When the beam angles are getting wider, the comparisons between the predictions and the experiment

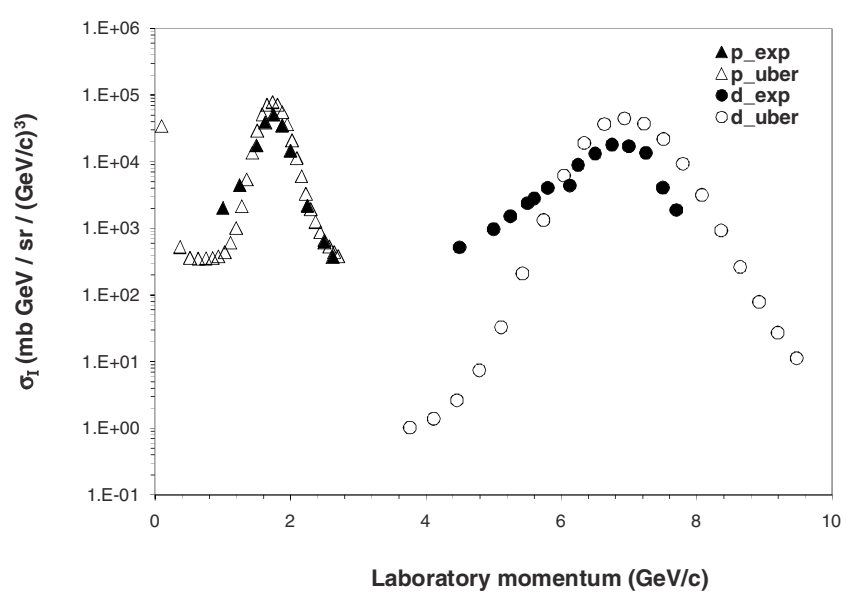

Fig. 2. The laboratory momentum spectrum at $2.5^{\circ}$ angle adapted from ref. [10] for the $1050 \mathrm{AMeV}{ }^{12} \mathrm{C}+{ }^{12} \mathrm{C}$ reaction compare with the predictions. The solid symbols represent experimental data and the open symbols are the model predictions with coalescence effects.

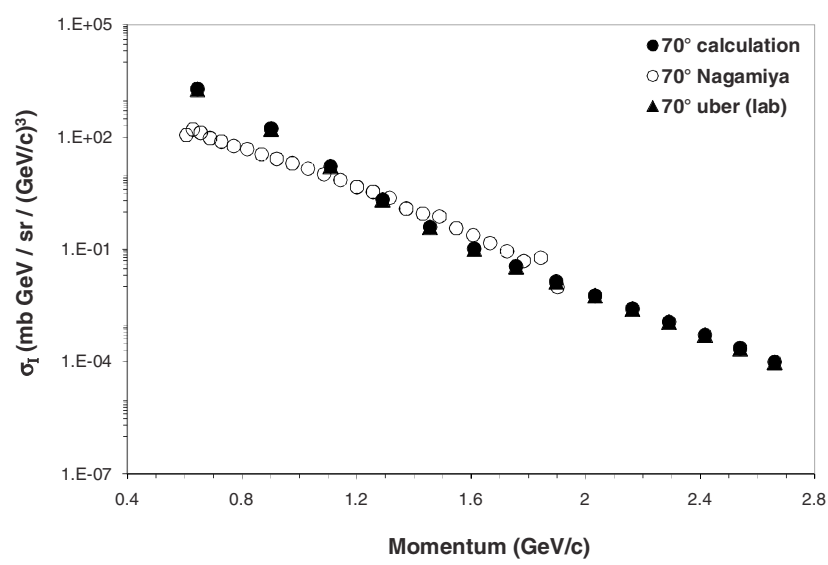

Fig. 3. Reported measurements from Nagamiya et al. [8] for deuteron invariant cross sections at $70^{\circ}$ in the laboratory frame from a ${ }^{12} \mathrm{C}$ beam colliding with a ${ }^{12} \mathrm{C}$ target. The deuteron invariant cross sections (solid circles) were calculated using Nagamiya's proton data and the coalescence model of ref. [8]. The deuteron invariant cross section predictions from the model described herein are displayed as solid triangles.

show stronger disagreement as shown in the $30^{\circ}$ angle plot. Figure 2 compares the experimental data at $1050 \mathrm{~A} . \mathrm{MeV}$ taken from ref. [10] with the proton invariant cross-section predictions from the code. The agreement demonstrates that the code presents an excellent prediction of the invariant cross sections for protons. The deuteron invariant cross section in the same figure shows a fairly good agreement. The results of including coalescence effects confirm the significant impact on the predicted cross sections (fig. 3). Figure 4 displays the predictions of alpha production from $800 \mathrm{~A} \cdot \mathrm{MeV}{ }^{12} \mathrm{C}$ beam interacting with ${ }^{12} \mathrm{C}$ nucleus. We are not aware of any available alpha distribution data from experiment to compare with at this time. 


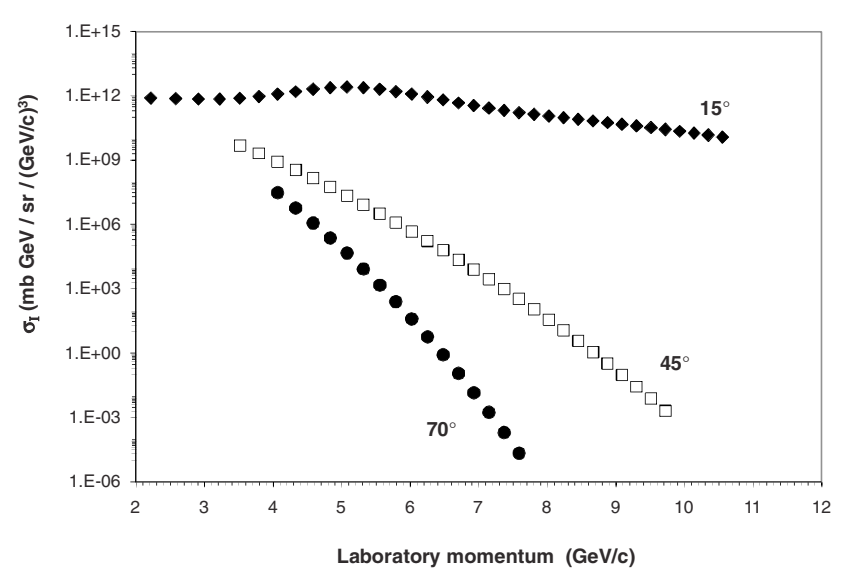

Fig. 4. The alpha particle distributions based on the predictions from the code are presented at various angles for a beam energy of $800 \mathrm{~A} \cdot \mathrm{MeV}{ }^{12} \mathrm{C}+{ }^{12} \mathrm{C}$.

\section{Conclusions and future work}

The two-stage abrasion-ablation model has been presented to describe the production of secondary neutrons. The coalescence effects are included in these collision formalisms. The model includes the contribution from both projectile and target nuclei. The predicted double differential neutron spectra at small forward angles agree well with the experimental data. However, there are some discrepancies at higher emission angles. The deuteron invariant cross sections at $800 \mathrm{~A} . \mathrm{MeV}$ ${ }^{12} \mathrm{C}+{ }^{12} \mathrm{C}$ reactions also show good agreement with the experimental data.
The results presented in this study clearly demonstrate the ability of the model to predict secondary neutron production and also the production of other light particles from both projectile and target nuclei. There are, however, some issues with the predictions at wider angles. Future work will focus on resolving these issues. Furthermore, the model will be extended to include nuclei of mass number greater than 68. It is also important to improve the computational time. Lastly, the future work will include more comparisons with the measurement data that are available.

\section{References}

1. F.A. Cucinotta, J.W. Wilson, L.W. Townsend, Nucl. Phys. A. 619, 202 (1997).

2. G.S. Braley et al., IEEE Trans. Nucl. Sci. 49, 2800 (2002).

3. S.T. Butler, C.A. Pearson, Phys. Rev. Lett. 7, 69 (1961).

4. A. Schwarzschild, Č. Zupančič, Phys. Rev. 129, 854 (1963).

5. J. Hüfner, K. Schäffer, B. Schürmann, Phys. Rev. C 12, 1888 (1975).

6. H.H. Gutbrod et al., Phys. Rev. Lett. 37, 667 (1976).

7. T.C. Awes et al., Phys. Rev. C 24, 89 (1981).

8. S. Nagamiya et al., Phys. Rev. C 24, 971 (1981).

9. T. Nakamura, L. Heilbronn, Handbook on Secondary Particle Production and Transport by High-Energy Heavy Ions (World Scientific, New Jersey, 2006), p. 224.

10. J. Papp, Single Particle Inclusive Spectra Resulting From the Collision of Relativistic Protons, Deuterons, Alpha Particles, and Carbon Ions with Nuclei, Ph. D. thesis, LBL-3633, 1975.

11. S Nagamiya et al., Lawrence Berkeley National Laboratory Report No. LBL-8463. 